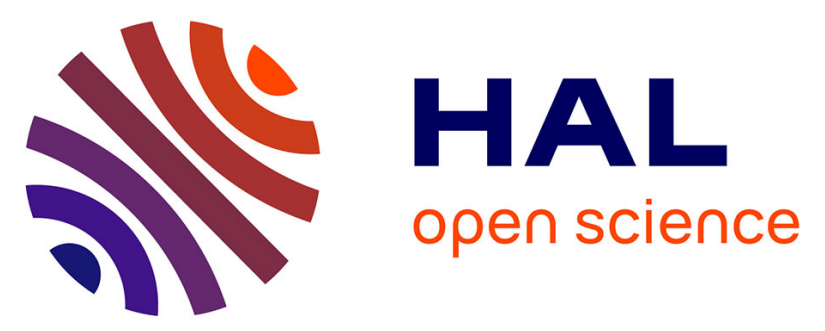

\title{
When the Giant meets the Ant An Asymmetric Approach for Collaborative and Concurrent Object Manipulation in a Multi-Scale Environment
}

Morgan Le Chénéchal, Jérémy Lacoche, Jérôme Royan, Thierry Duval, Valérie Gouranton, Bruno Arnaldi

\section{To cite this version:}

Morgan Le Chénéchal, Jérémy Lacoche, Jérôme Royan, Thierry Duval, Valérie Gouranton, et al.. When the Giant meets the Ant An Asymmetric Approach for Collaborative and Concurrent Object Manipulation in a Multi-Scale Environment. 3DCVE 2016: International Workshop on Collaborative Virtual Environments, Mar 2016, Greenville, United States. pp.1 - 4, 10.1109/3DCVE.2016.7563562 . hal-01293041

\section{HAL Id: hal-01293041 \\ https://hal.science/hal-01293041}

Submitted on 24 Mar 2016

HAL is a multi-disciplinary open access archive for the deposit and dissemination of scientific research documents, whether they are published or not. The documents may come from teaching and research institutions in France or abroad, or from public or private research centers.
L'archive ouverte pluridisciplinaire HAL, est destinée au dépôt et à la diffusion de documents scientifiques de niveau recherche, publiés ou non, émanant des établissements d'enseignement et de recherche français ou étrangers, des laboratoires publics ou privés. 


\section{When the Giant meets the Ant An Asymmetric Approach for Collaborative and Concurrent Object Manipulation in a Multi-Scale Environment}

\author{
Morgan Le Chénéchal * \\ IRT $b<>$ com \\ Thierry Duval ${ }^{\dagger}$ \\ Télécom Bretagne / Lab-STICC \\ IRT $\mathrm{b}<>$ com
}

\author{
Jérémy Lacoche * \\ IRT $\mathrm{b}<>$ com \\ Valérie Gouranton * \\ INSA Rennes / Irisa/INRIA \\ IRT $\mathrm{b}<>$ com
}

\author{
Jérôme Royan * \\ IRT $b<>$ com \\ Bruno Arnaldi $\ddagger$ \\ INSA Rennes / Irisa/INRIA \\ IRT $b<>$ com
}

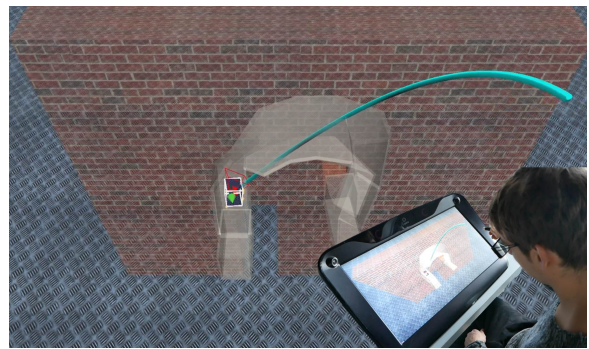

(a)

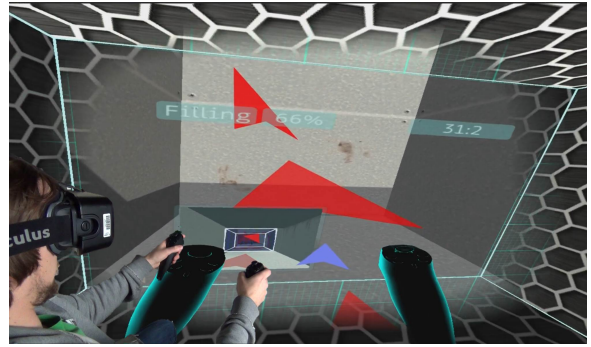

(b)

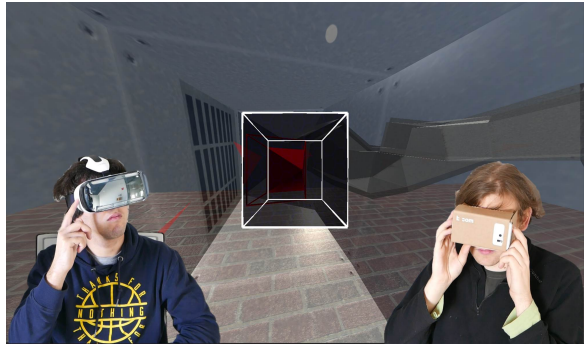

(c)

Figure 1: Collaborative manipulation of a virtual object (here, a cube) based on an asymmetric setting between two users who can be helped by two additional users. (a) The first participant has a global view of the scene and moves the object with a 3D bent ray. (b) The second user is placed inside the object and precisely rotates and scales it. (c) Two additional roles can be added. The first one helps to scale the object using a third person view of it. The other one is a spectator who switches between the other participants' viewpoints and helps them with oral communication.

\begin{abstract}
In this paper, we propose an innovative approach that enables two or more users to manipulate an object collaboratively. Our goal is to benefits from the wide variety of todays VR devices. Therefore, our solution is based on an asymmetric collaboration pattern at different scales in which users benefit from suited points of views and interaction techniques according to their device setups. Indeed, each user application is adapted thanks to plasticity mechanisms. Our system provides an efficient way to co-manipulate an object within irregular and narrow courses, taking advantages of asymmetric roles in synchronous collaboration. Moreover, its aims to provide a way to maximize the filling of the courses while the object moves on its path.
\end{abstract}

Keywords: Collaborative 3D Interactions ; Shared Virtual Environments

Index Terms: H.5.3 [Information interfaces and presentation (e.g HCI)]: Group and Organization Interfaces-Computer supported cooperative work (CSCW); I.3.6 [Computer Graphics]: Methodology and Techniques-Interaction techniques

\footnotetext{
*e-mail:\{morgan.lechenechal,jeremy.lacoche,jerome.royan\} @b-com.com

†e-mail: thierry.duval@telecom-bretagne.eu

¥e-mail: \{valerie.gouranton,bruno.arnaldi\} @ irisa.fr
}

\section{INTRODUCTION}

In this paper, we propose a set of metaphors that enables several users to collaborate in a shared multi-scale Virtual Environment (VE) [7, 12] in order to achieve collaborative manipulation tasks in Virtual Reality (VR). Such tasks could consist in overcoming different obstacles by moving, rotating and scaling an object collaboratively. This kind of collaborative manipulation can be used to simulate industrial tasks such as in an automotive factory where cumbersome objects must be carried by several collaborators [1]. To do so, we propose an asymmetric collaboration between two or more users with different devices (cf. Fig. 11. These users are embedded in a co-located multi-scale VE thanks to a model inspired from the Immersive Interactive Virtual Cabin (IIVC) generic model [5].

This work is motivated by the current state of the VR market. All devices used are or will be soon on the consumer market: a zSpace, an Oculus Rift with a Razer Hydra, a Gear VR and a Google Cardboard. Indeed, today there is growing number of available VR visualization and interaction devices. Each setup does not offer the same interaction capabilities. That is why our approach benefits from this diversity by proposing a set of interaction techniques adapted to different device setups in order to perform a collaborative manipulation task. These techniques are automatically associated to the suited users with plasticity mechanisms. Plasticity is defined as the capacity of an interactive system to withstand variations of both the system physical characteristics and the environment while preserving its usability [18]. Here, we demonstrate an example of plasticity for device and collaboration adaptations thanks to the models presented by Lacoche et al. [8].

As proposed by Pihno et al. [13], our approach splits the Degrees 
of Freedom (DoFs) of the manipulated object between collaborators in order to maximize the system efficiency: the Giant (with a global viewpoint) controls the object's translation, while the Ant (inside the object) sets its scale and rotation. In our shared multi-scale VE, this approach allows the Giant to quickly move the object, while the Ant performs better accurate transformations and has a suited viewpoint to maximize the courses filling. The object's rotation can also be shared between the two main users using a particular fusing scheme inspired from the asymmetric integration by Ruddle et al. [16]. In this case, we use a non-linear merging factor for the Giant's action according to its ray bending. Last, the third helping user (with the $3^{\text {rd }}$ person viewpoint of the object) can also set the scale of the manipulated object by asymmetrically integrating its action with the Ant's one. All metaphors for controlling these DoFs are designed to have an easy and quick learning curve for the end user.

In order to demonstrate the interest and the efficiency of our solution, we have proposed to test it in the context of the IEEE 3DUI contest 2016. The goal of this contest was to move a cube collaboratively through different labyrinths, while maximizing the courses filling of the object. Most examples given in this paper are based on the manipulation tasks required by this contest.

This paper is structured as follows: first, an overview of this work is presented in Section 2 Second, we present collaborators' roles of our proposal in Section 3 then we detail interaction techniques in Section 4 . Section 5 describes our implementation and Section 6 discusses informal feedbacks of test users. Last, we conclude and present perspectives of this work in Section 7

\section{OVERVIEW}

In this work, referring to the classification by Margery et al. [11], we focus on cooperation at level 3 . We want that the different users can change the same object transformation at the same time. This kind of synchronous collaborative object manipulation in CVE can be divided in two families: symmetrical and asymmetrical approaches. In the first symmetrical approach, collaborators benefit from equivalent viewpoints and interaction capabilities. This collaboration can be homogeneous based on 3D ray [14] or 3D pointer (i.e virtual hands metaphor) [4, 1], or heterogeneous, for instance mixing a 3D ray for a user, and a 3D pointer for another one [13]. On the contrary, in asymmetrical approaches, the users collaborate in a multi-scale CVE, i.e they have different viewpoints, thus their interaction techniques must be adapted to their different capabilities [3] 5

Both families can be implemented in two different ways: split of DoFs (level 3.1) or concurrent modification involving a merge policy (level 3.2). In our work, we use both conditions in order to maximize the efficiency of the whole proposed approach. Basically, the object manipulation uses a split of DoFs policy between the Giant and the Ant. However, in some conditions, the Giant can take the lead on the Ant rotation capability in order to help him. This is done by merging both inputs with a custom policy giving a bigger influence to the Giant action. Then, if a third user joins the CVE, the scale of the object is controlled using a cooperative interaction with the Ant at level 3.2. We did not find any related work that uses this kind of adaptable approach. Moreover, our proposal involves a kind of hybrid manipulation/navigation technique for the Ant. Indeed, the Ant is placed inside the manipulated object, moved by the Giant. This feature involves the Ant's navigation in the CVE, controlled by the Giant. However, the Ant does not control the manipulated object location, thus, the approach does not require any navigation technique even if the Ant may feel as in a car or a spaceship. This hybrid approach is also an innovative way to apprehend co-manipulation using an asymmetrical approach to improve the efficiency of this difficult task.

\section{Asymmetric COLLABORATIVE SCEnARIO}

We propose an asymmetric collaboration where each user benefits from interaction capabilities adapted to his interaction devices in order to move, rotate and scale a virtual object. It is based on two main roles, a Giant with a global view of the shared environment, and an Ant inside the manipulated object. Two other roles are also possible for assisting the two main users.

\subsection{Global View: the Giant}

The first user is interacting on a zSpace ${ }^{1}$ as shown in Figure $1 \mathrm{a}$ It is composed of a 3D stereoscopic display with head tracking and of a 3D tracked stylus for interacting. The zSpace screen is used to create a window to the VE. Therefore, this user has a global view of the scene and can roughly manipulate the object in order to move it really fast in easy passages. This user can translate the object and also shares with the Ant the possibility to rotate it.

\subsection{Micro View: the Ant}

The second user visualizes the scene with a Head-Mounted Display (HMD), here an Oculus Rif ${ }^{2}$ as shown in Figure $1 \mathrm{~b}$ He is interacting with a Razer Hydre 3 composed of two 3D tracked controllers. We exploit the immersion feeling given by the HMD to place this user inside the manipulated object. This position enables him to manipulate the object with a fine accuracy. His role is essential to overcome difficult passages and maximize the courses filling by the object. He can scale the object and shares the possibility to rotate it with the Giant. His scale in the scene also offers him direct interaction possibilities such as pushing buttons to trigger different actions.

\subsection{Third Helping User}

As shown in Figure $1 \mathrm{c}$, the third user is interacting with a GearVR 4 an HMD with a 2D trackpad. His role is optional. This user has a third person view of the manipulated object. His role is to help the Ant to scale it with slide gestures on the GearVR trackpad. Therefore, the scaling capability is shared by these two users.

\subsection{Spectators}

The last role is a spectator. It is available on multiple devices. In our scenario, he uses a Google Cardboar 5 as shown in Figure $1 \mathrm{c}$ Multiple spectators can be included in the shared VE. These users can switch between the other participants' viewpoints. Here, it is done by pulling the Cardboard trigger. They can help the other participants by giving oral instructions.

\section{INTERACTION TECHNIQUES}

The collaborators benefit from complementary interaction techniques to perform collaborative manipulations task that need translating, rotating, and scaling. An example of task is given in Figures $1 \mathrm{a}$ 1b and 1c This task is one of the tasks required by the IEEE 3DUI contest 2016. The goal is to pass a cube through a labyrinth while maximizing the courses filling of the object. First, we present the Giant's interation technique based on a bentray in Subsection 4.1 Then, we describe the Ant's interaction technique in Subsection 4.2 and the handling of optional concurrent manipulation (for the object rotation and scale) in Subsection 4.3

$$
\begin{aligned}
& \sqrt[1]{\text { http://zspace.com/ }} \\
& \sqrt[3]{\text { https://www.oculus.com/en-us/ }} \\
& 3_{\text {http://www.razerzone.com/fr-fr/ }}^{\text {gaming-controllers/razer-hydra-portal-2-bundle }} \\
& 4^{4} \text { http://www.samsung.com/fr/galaxynote4/gear-vr/ } \\
& \text { https://www.google.com/get/cardboard/ }
\end{aligned}
$$




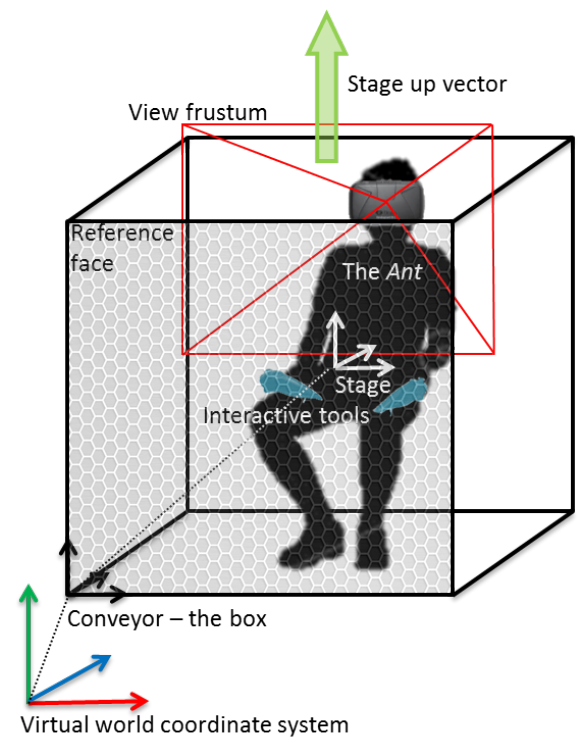

(a)

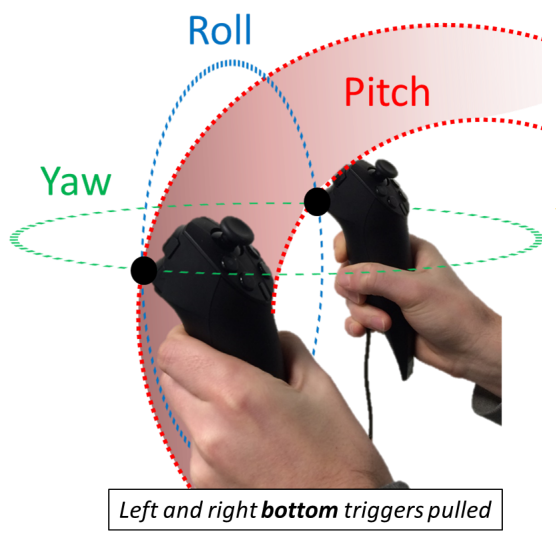

(b)

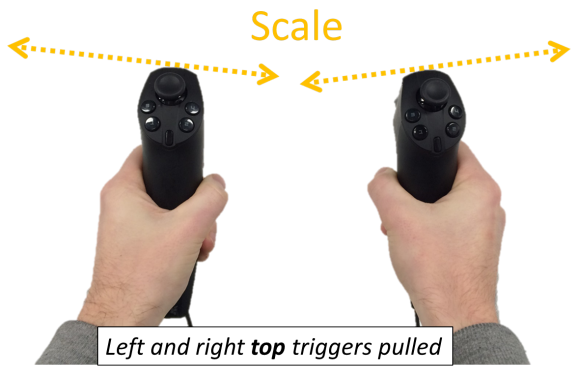

(c)

Figure 2: (a) The Ant's IIVC instantiation. The front textured face of the object (here, a box) is the current stage front face that is used as reference for object manipulation performed by the Ant. The stage coordinate system is always centered in the object. (b) and (c) The two metaphors used by the Ant to rotate and scale the manipulated object.

\subsection{Global Manipulation}

The user on the zSpace (Giant) can translate the object (actually the Ant's conveyor in the IIVC model) with a bent ray inspired from the interaction technique proposed by Riege et al. [15]. The ray is controlled in position and rotation by the tracked stylus. One button is used for object grabbing, and the other buttons are used to switch between four point of views: front, left, back and right. The ray is bent during the object translation in order to respect three constraints:

- First, the manipulated object is attached to the ray extremity with a spring joint, as proposed by Fröhlich et al. [6]. The physical collider of the object avoids it to pass through other objects. The ray is bent accordingly.

- Second, we manually limit the ray extremity speed when an object is grabbed. The goal is to not disturb the distant user inside the manipulated object and reduce his cybersickness.

- Third, a last constraint is optional. We added an active help for the translation. It is a magnetic path that represents the perfect path to follow. The manipulated object is also connected to the closest point on this path with a spring joint.

To make the others understand the Giant's actions, his head, stylus and 3D ray are rendered in the shared environment as shown on the top Figure $5 \mathrm{a}$

\subsection{Inside Object Manipulation}

The Ant is placed inside the manipulated object. He can scale and rotate it with the two Razer Hydra controllers thanks to bimanual metaphors inspired from the work of Cutler et al. [2]. These manipulations are performed with a fix reference: the object front face. This reference face can be changed. Figure 2 a illustrates our instantiation of the IIVC model for the Ant, and especially explains the meaning of the reference face in the user stage according to the manipulated object. We propose a symmetric technique between both hands to switch the current stage front face based on joysticks. Up, bottom, right and left joystick triggers are used to apply a $90^{\circ}$ rotation to the stage. An asymmetric aspect is introduced in order to turn around the normal current front face. For this purpose, we use the joystick button capability, to choose if we turn in the clockwise or the counter-clockwise direction, according to the hand used.

Manipulations of the conveyor are physically constrained, thus, the manipulated object can not pass through an obstacle. As shown in Figure $2 b$ the rotation is made with a modified version of the grab-and-twirl metaphor. Compared to the classical version, the pitch rotation is performed with a metaphor close to a plane yoke by orienting the two controllers to the top or to the bottom. The scale of the object is controlled with a grab-and-scale metaphor by bringing closer or further the two Razer Hydra controllers while pushing two corresponding buttons (cf. Fig 2c). According to the selected mode, the object scale can be defined as uniform, based on the distance between the two controllers, or non-uniform by projecting the distances on the $\mathrm{x}, \mathrm{y}$ and $\mathrm{z}$ axis of the Ant's stage.

Two visual feedbacks are rendered to make the Ant understand the distance between the manipulated object and possible obstacles. First, we render particles at the collision points. Second, a virtual grid visible in blue at bottom in Figure $1 \mathrm{~b}$, parallel to the user current front face, is displayed outside of the object. In addition to these feedbacks, we compute a filling ratio that is displayed in the integrated Graphical User Interface (GUI). It allows the Ant to be aware of the courses filling by the manipulated object, and to maximize it by using this quantitative ratio as well as the $3 \mathrm{D}$ visual feedbacks.

In some particular situations, local interactors can be integrated at scale one in the VE. For instance, in the example given in Figure $5 \mathrm{~b}$, buttons are available in the scene for triggering different actions such as opening doors. These buttons are too smalls for the Giant. Therefore, as the two Razer Hydra controllers are rendered into the VE, the Ant can use them to interact with these local interactors. Here, by touching one button with one of the controller, the Ant can trigger the corresponding action.

To guide the Ant when he is placed in a closed environment such as the provided labyrinth, different spatial cues can help him. They are shown in Figure 1b First, a World-In-Miniature [17] shows a third person view that focuses on the manipulated object. Second, 
Figure 3: Scheme of the technique used to apply rotation to the object using the Giant's bentray. $A$ and $B$ are respectively the beginning and the end of the straight, not rendered, ray. $C$ is the end of the rendered bentray. Axis of rotation is computed as the cross product between $\overrightarrow{A B}$ and $\overrightarrow{B C}$.

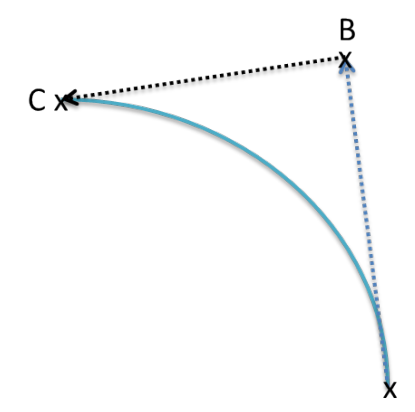

A

an arrow simulates a compass to show him the direction to follow. Third, in the labyrinth, the path to follow is also indicated with arrow signs.

As shown in Figure $1 \mathrm{~b}$ a progressive transparency effect is applied to the manipulated object from the screen extremities to the screen center. This technique is adapted from the proposal described in [9]. Here, it is used as an anti-cybersickness filter that aims to make the peripheral view of the user consistent with his head movement. Therefore, the user's peripheral view is less disturbed by translations performed by the Giant. Some preliminary evaluations of this effect have been performed in another context and have shown good results.

For awareness issues, the viewpoint of the Ant is shown to the other users by displaying his frustum and stage up vector.

\subsection{Concurrent Manipulation}

As optional collaboration possibilities, we provide concurrent manipulation capabilities for the rotation and the scale of the object.

\subsubsection{Rotation}

We propose an optional rotation control scheme based on the concurrent action of the Giant and the Ant. In some particularly difficult circumstances, for instance if the Ant is completely lost and does not achieve to find the correct way to orient the object, the $\mathrm{Gi}$ ant can help him to rotate the object by acting on the Ant's conveyor orientation. For this purpose, the Giant can use his bentray-based interaction technique as explained in scheme Fig 3 The interaction starts when the bending of the ray is up to a specific threshold, i.e when $\overrightarrow{B C}$ length is up to $2.0 \mathrm{~m}$ in our implementation. Then, the manipulated object rotation is controlled based on velocity according to the $\overrightarrow{B C}$ length, as follow:

Each frame

if $\overrightarrow{B C}$.length $>$ threshold then

angle $=((\overrightarrow{B C} \cdot \text { length }- \text { threshold }) * \text { velocity } * \text { deltaTime })^{2}$

end if

object.rotateAround $(\overrightarrow{A B} \times \overrightarrow{B C}$, angle $)$

It results in a concurrent manipulation with the rotation capability of the Ant that is performed by integrating both actions with a growing Giant's factor according to its ray bending.

\subsubsection{Scale}

The scale control is shared between the Ant and the user of the GearVR. To solve this concurrency, we add the factors that the two users want to apply to the scale. Thus, the fuse of the concurrent scale control is not based on an average method, but on a relative setting that allows synchronous manipulation without conflict. It means that each frame, each user asks to the scale manager component to increase or decrease the object scale by a vector. Then, this manager adds all the requests and sets the object scale accordingly if possible (i.e taking care of environmental physical constraints).

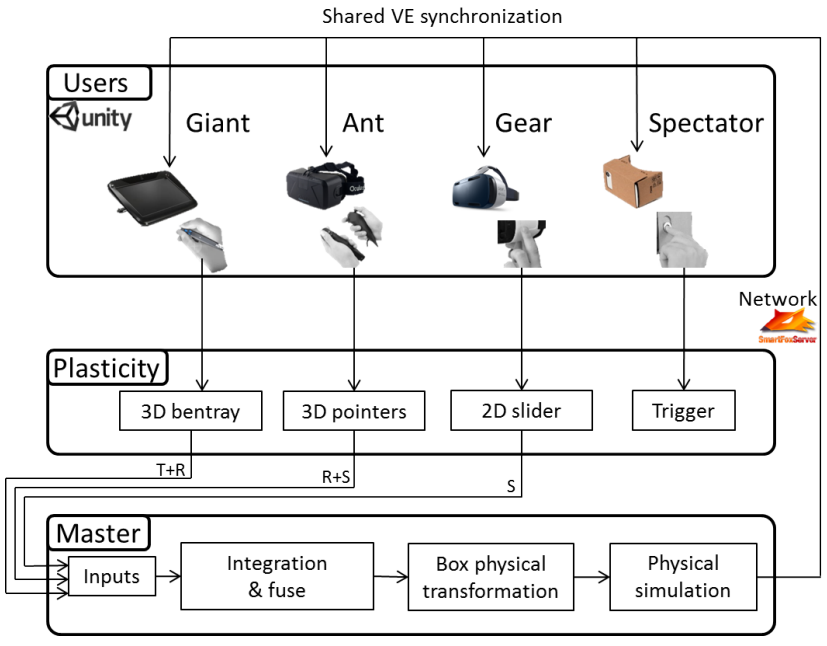

Figure 4: The global architecture of our implementation. T,R and S respectively refer to translation, rotation and scale.

\section{IMPLEMENTATION}

Regarding the implementation of the prototype, Figure 4 illustrates the architecture of the application. A software overlay of SmartFox Server ${ }^{6}$ is used to manage collaboration. It ensures the synchronization of the shared VE between the different users as well as a consistent physical simulation. For rendering, scripting and managing the scene we use the Unity3D game engine 7 The interaction part is independent from the devices and from the game engine used. Indeed, it is developed with an implementation of the plasticity models presented by Lacoche et al. [8]. With this solution, each user automatically benefits from the adapted interaction techniques according to his available devices. These plasticity mechanisms also give us the possibility to easily exchange the current devices used. For instance, we could use an HTC Vive 8 instead of the combination Oculus Rift / Razer Hydra for the Ant or any desktop environment instead of the Google Cardboard for the spectator.

\section{Preliminary Tests}

We did not perform any formal evaluation. However, we tested our approach with different users and scenarios. These first test users have experience with VR applications and 3D interactions. Regarding the commands of the Ant, we first tried the classical version of the grab-and-twirl metaphor in order to modify the manipulated object rotation. The different users did not feel comfortable with this interaction and they seemed to perform better with our interaction technique that reminds a plane yoke. Indeed, as the user is placed inside the object, controlling it as the user would control a vehicle seemed more natural. To continue, the different visual feedbacks for the Ant for making him understand his spatial relationship with the environment were added after multiple tests. Indeed, as the user did not known well the different labyrinths, they seemed to bo relatively lost after multiple movements. For the God, we have compared the bent ray with a classical straight one without any speed limitation. This approach was really easy to understand for the God but it was really disturbing for the Ant to move as fast. Moreover, when the Ant was translated too fast, he did not have the time to anticipate the next obstacles and adapt his rotation.

\footnotetext{
6htp: //www.smartfoxserver.com/

https://unity3d.com/

8 https://www.htcvive.com/us /
} 


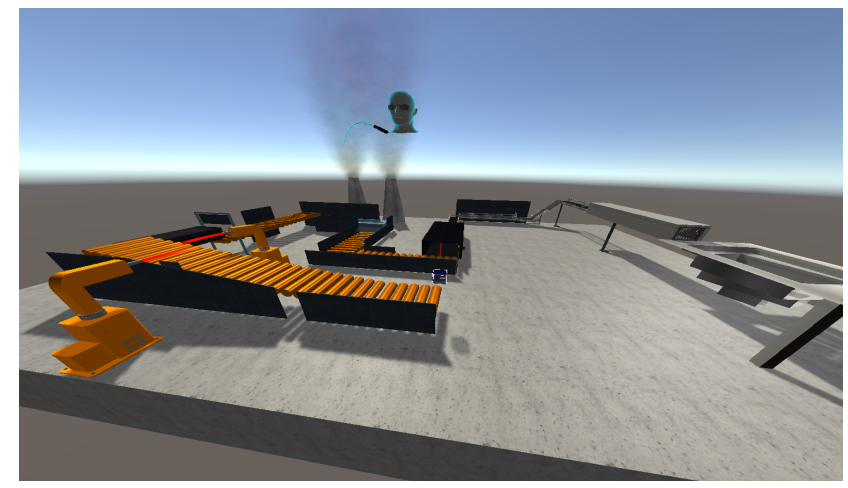

(a)

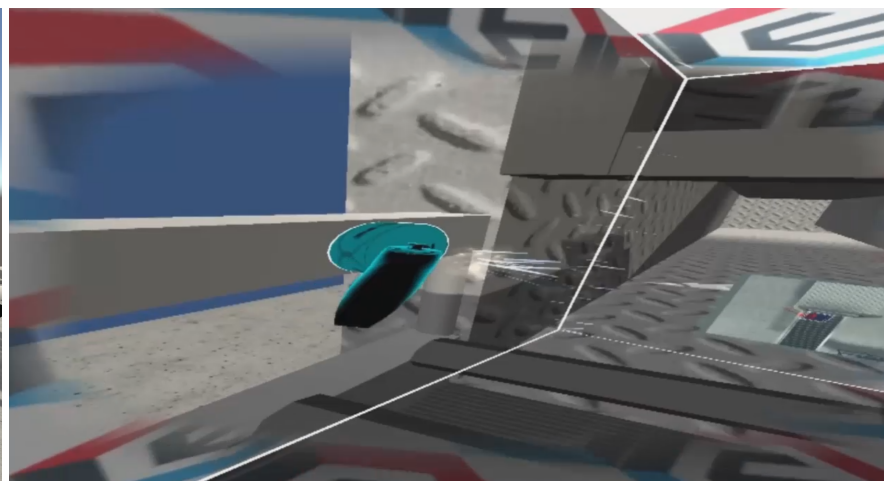

(b)

Figure 5: The virtual factory scene that provides scale-one interactions. Buttons are present in the scene to trigger different actions such as opening doors or painting the object. Triggering these actions is needed to fulfill the task. Therefore, as shown on (b), the Ant has direct manipulation capabilities, he can push a button by colliding it with one of his controllers.

\section{Conclusion \& Perspectives}

In this paper, we proposed an asymmetric approach for comanipulation in shared multi-scale environment. It is based on the collaboration between a Giant with a global view of the scene and an Ant immersed inside the manipulated object. Additional users can also be included to help the two main users in their task completion. Moreover, our approach benefits from a plasticity mechanisms that handle the automatic adaptation of the interaction technique according to the device used by the different users with heterogeneous setups.

This work has been proposed to complete the different tasks required by the IEEE 3DUI contest 2016. An illustrative video has also been published with the short descriptive paper [10].

Preliminary users tests show a good efficiency of the different interactions techniques. A formal evaluation should be done in order to confirm the performances of the approach. First, it would be interesting to compare our proposal with a solution from the state-of the art where the collaboration is symmetrical, i.e with equivalent roles and viewpoints. Second, our solution includes an oral communication between the different participants. It would be interesting to evaluate the set of interaction techniques without this oral communication. As a result, we could include other awareness mechanisms in order to improve the collaboration when the participants can not talk with each other.

\section{References}

[1] L. Aguerreche, T. Duval, and A. Lécuyer. Short paper: 3-hand manipulation of virtual objects. In JVRC 2009, pages 4-p, 2009.

[2] L. D. Cutler, B. Fröhlich, and P. Hanrahan. Two-handed direct manipulation on the responsive workbench. In Proceedings of the 1997 symposium on Interactive $3 D$ graphics, page 107114, 1997.

[3] T. Duval and C. Fleury. An asymmetric $2 \mathrm{~d}$ pointer/3d ray for $3 \mathrm{~d}$ interaction within collaborative virtual environments. In Proceedings of the 14th international Conference on $3 D$ Web Technology, pages 33-41. ACM, 2009.

[4] T. Duval, A. Lécuyer, and S. Thoma. Skewer: a 3d interaction technique for 2-user collaborative manipulation of objects in virtual environments. In 3D User Interfaces, 2006. 3DUI 2006. IEEE Symposium on, pages 69-72. IEEE, 2006.

[5] C. Fleury, A. Chauffaut, T. Duval, V. Gouranton, and B. Arnaldi. A Generic Model for Embedding Users' Physical Workspaces into Multi-Scale Collaborative Virtual Environments. In ICAT 2010, Adelaide, Australia, Dec. 2010.
[6] B. Fröhlich, H. Tramberend, A. Beers, M. Agrawala, and D. Baraff. Physically-based manipulation on the responsive workbench. In $V R$ 2000. Proceedings. IEEE, pages 5-11. IEEE, 2000.

[7] H. Ibayashi, Y. Sugiura, D. Sakamoto, N. Miyata, M. Tada, T. Okuma, T. Kurata, M. Mochimaru, and T. Igarashi. Dollhouse vr: a multi-view, multi-user collaborative design workspace with vr technology. In $S I G-$ GRAPH Asia 2015 Emerging Technologies, page 8. ACM, 2015.

[8] J. Lacoche, T. Duval, B. Arnaldi, E. Maisel, and J. Royan. Plasticity for 3D User Interfaces: new Models for Devices and Interaction Techniques. In EICS 2015. ACM.

[9] J. Lacoche, M. Le Chénéchal, S. Chalmé, J. Royan, T. Duval, V. Gouranton, É. Maisel, and B. Arnaldi. Dealing with frame cancellation for stereoscopic displays in 3d user interfaces. In 3DUI 2015, pages 73-80. IEEE, 2015.

[10] M. Le Chénéchal, J. Lacoche, J. Royan, T. Duval, V. Gouranton, and B. Arnaldi. When the Giant meets the Ant: An Asymmetric Approach for Collaborative Object Manipulation. In $3 D$ User Interfaces (3DUI), 2016 IEEE Symposium on. IEEE, 2016.

[11] D. Margery, B. Arnaldi, and N. Plouzeau. A general framework for cooperative manipulation in virtual environments. Springer, 1999.

[12] T. T. H. Nguyen, C. Fleury, and T. Duval. Collaborative exploration in a multi-scale shared virtual environment. In $3 D$ User Interfaces (3DUI), 2012 IEEE Symposium on, pages 181-182. IEEE, 2012.

[13] M. S. Pinho, D. A. Bowman, and C. M. Freitas. Cooperative object manipulation in immersive virtual environments: framework and techniques. In Proceedings of the ACM symposium on Virtual reality software and technology, pages 171-178. ACM, 2002.

[14] M. S. Pinho, D. A. Bowman, and C. M. Freitas. Cooperative object manipulation in collaborative virtual environments. Journal of the Brazilian Computer Society, 14(2):54-67, 2008.

[15] K. Riege, T. Holtkamper, G. Wesche, and B. Fröhlich. The bent pick ray: An extended pointing technique for multi-user interaction. In 3DUI 2006, pages 62-65, March.

[16] R. A. Ruddle, J. C. Savage, and D. M. Jones. Symmetric and asymmetric action integration during cooperative object manipulation in virtual environments. ACM Transactions on Computer-Human Interaction (TOCHI), 9(4):285-308, 2002.

[17] R. Stoakley, M. J. Conway, and R. Pausch. Virtual reality on a WIM: interactive worlds in miniature. In Proceedings of the SIGCHI conference on Human factors in computing systems, page 265272. ACM Press/Addison-Wesley Publishing Co., 1995.

[18] D. Thevenin and J. Coutaz. Plasticity of user interfaces: Framework and research agenda. In Proceedings of INTERACT, volume 99, page 110117, 1999. 